\title{
Global task force for influenza
}

\author{
Early detection and rapid response to bird flu, on a global scale, will drastically cut the costs of dealing with a \\ full-blown human flu pandemic, argue Ron Fouchier, Thijs Kuiken, Guus Rimmelzwaan and Albert Osterhaus.
}

A human flu pandemic could cause $20 \%$ of the world's population to become ill. Within a few months, close to 30 million people would need to be hospitalized, a quarter of whom would die ${ }^{1}$. Although these estimates are speculative, they are among the more optimistic predictions of how the next flu pandemic might unfold.

Like most emerging virus infections that threaten human health, flu outbreaks originate from animal reservoirs. Because of rapidly changing human behaviour and animal ecol$\mathrm{og}^{2}$, infections are spreading faster and farther. Patchy research on these outbreaks, and poor coordination between different disciplines in response to them, are limiting our ability to deal adequately with the threat they pose to human health. We propose establishing a permanent global task force to control a flu pandemic, in which relevant agencies would work together with leading research groups from different disciplines.

\section{Close watch}

An outbreak of avian flu among chickens in the Netherlands in 2003 led to the culling of 31 million birds. Initially, the ministry of health issued warnings, but the ministry of agriculture insisted that there was no risk to human health. "I've worked with sick chickens all my life and never become ill before," was the sentiment commonly voiced by veterinarians involved in the culling activities. But by the end of the outbreak, 89 people were confirmed to be infected with the flu virus strain H7N7; one died and the rest experienced eye disease or common flu symptoms ${ }^{3}$.

In southeast Asia, several countries are currently affected by the H5N1 strain of flu virus. Animal health authorities in some countries have delayed reporting the disease in poultry, in some cases by several weeks, and this has contributed to the failure to contain these epidemics. Such delays have important implications for human health; most human cases of avian flu to date have been linked to contact with infected poultry.

Traditionally, the poultry industry has approached avian flu as an economic problem. Poultry are tested only when animals become sick and die, and even then testing is generally restricted to a search for highly pathogenic strains of the $\mathrm{H} 5$ and $\mathrm{H} 7$ flu virus subtypes, excluding the other 14 subtypes and less pathogenic strains. Yet the past three human flu pandemics did not originate from $\mathrm{H} 5$ or $\mathrm{H} 7$ viruses ${ }^{4}$. Moreover, the reporting of viral infections is often delayed because of authorities' conflicting mandates to report disease

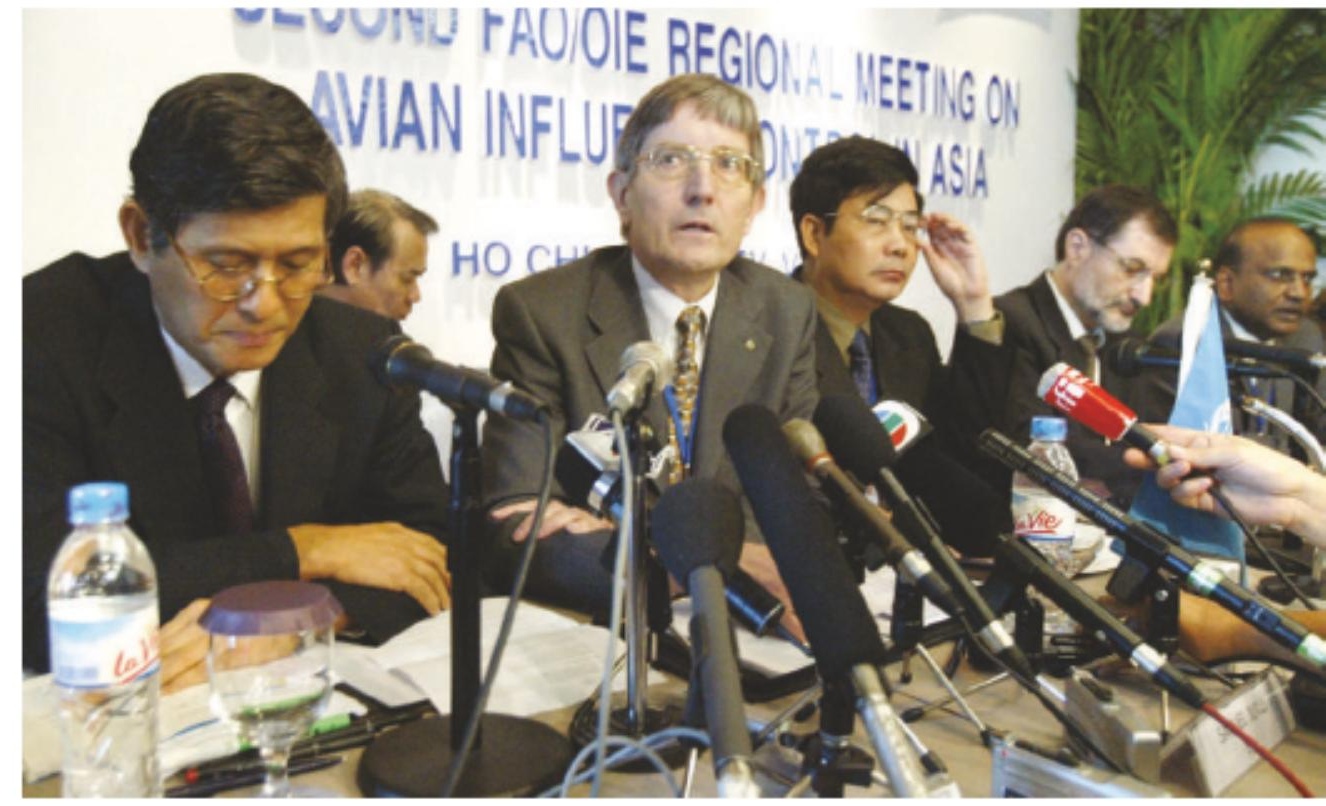

Pulling together: agency cooperation is es sential to prepare for the threat posed by avian flu.

occurrences and to protect their country's export status for animal products.

At the international level, trade regulations for poultry and poultry products are defined by the World Organization for Animal Health (OIE) based in Paris. However, there is geographical variation in production and distribution systems for poultry (for example, farms may mix species, or have open access to wild birds, birds may be taken live to market or killed beforehand). The extent to which affected countries follow effective outbreakcontainment measures is also variable; culling and vaccine-deployment strategies are not standardized. And measures to protect poultry workers during flu outbreaks vary greatly from country to country.

This lack of international harmony in detecting and dealing with avian flu extends to the assessment of human infections. On the basis of 51 deaths out of 88 laboratory-confirmed cases ${ }^{5}$, the fatality rate from $\mathrm{H} 5 \mathrm{~N} 1 \mathrm{flu}$ virus infection in humans is estimated to be around $60 \%$. But the true incidence of infection, and the associated spectrum of disease symptoms, may be very different ${ }^{6}:$ data on post-mortem investigations are largely lacking, for example, limiting our knowledge of which tissues the virus attacks and how it causes disease. It is therefore impossible to determine which of the available and very variable (in terms of their pathological outcome) animal models - mouse, ferret, cat, pig, macaque - most closely resembles the disease in humans.
Specific monitoring of virological, serologi$\mathrm{cal}$ and clinical parameters is urgently needed for people at risk. Also needed are detailed autopsies to characterize the disease, and the subsequent establishment of appropriate animal models to evaluate available intervention strategies. For poultry, bird populations should be actively surveyed for all subtypes of flu virus, using high-throughput technology; production and distribution systems should be modified; and stricter adherence to containment measures achieved when there is an outbreak.

\section{Integration}

To obtain a better global picture of the threat posed by avian flu, it is imperative to investigate the virus in wild bird populations. Wild birds, particularly migratory ducks, geese and shorebirds, are the natural reservoir of influenza A viruses, which can infect other avian and mammalian species ${ }^{7}$. But information about flu in wild birds is still limited. A widespread and integrated approach is needed to understand the dynamics, epidemiology and pathogenesis of these virus infections in wild birds, and the potential routes of virus transmission. For example, transmission from wild birds to poultry and mammalian species, including humans, may result from direct contact with production systems, the wild bird trade, and smuggling.

To limit the effects of flu on public health and livestock production, integrated and effective action from all the disciplines 


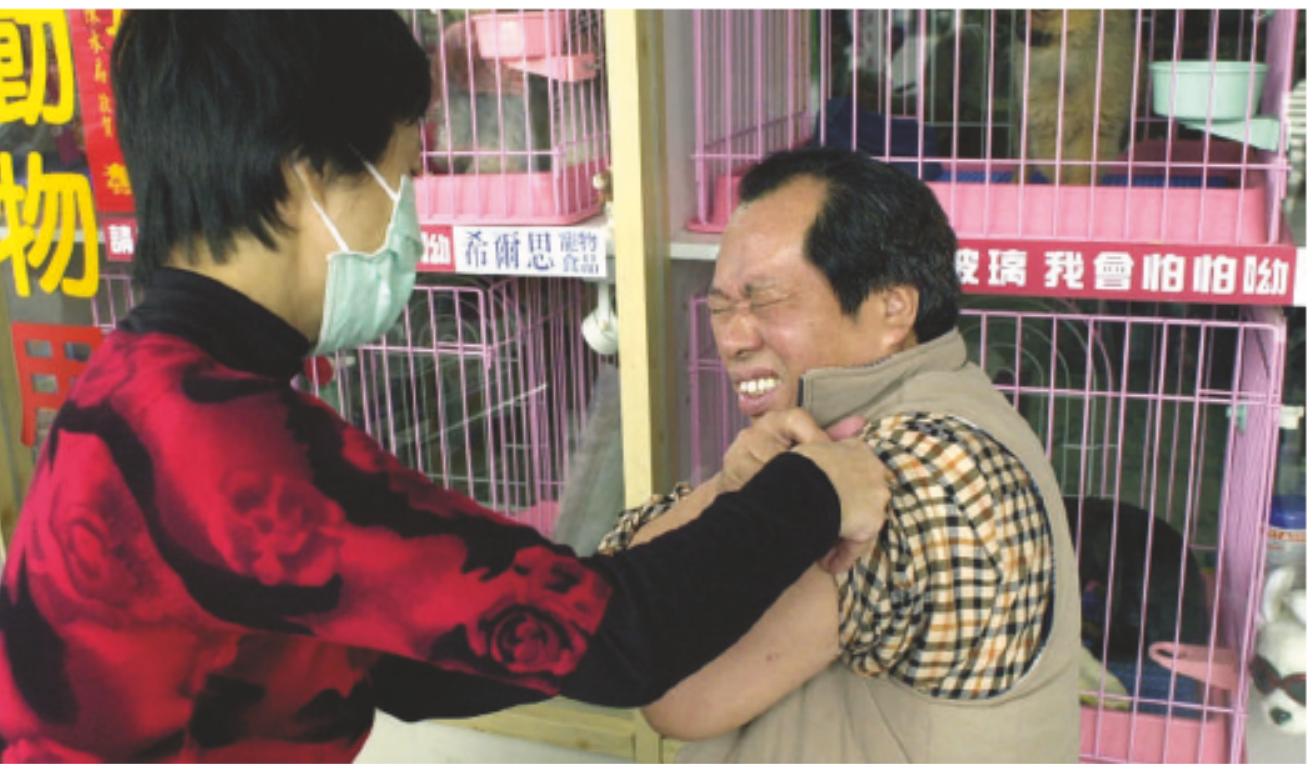

Group effort: to tackle flu effectively, greater coordination is needed between the health workers providing vaccinations (above) and the veterinarians monitoring poultry (below).

involved is urgently needed, rather than ad-hoc responses at the national level. To this end, we advocate a global flu task force as an essential element of the World Health Organization's (WHO's) flu pandemic preparedness plan.

\section{Task-force agenda}

This task force should consist of leading specialists in the fields of human and animal medicine, virology, epidemiology, pathology, ecology and agriculture, as well as experts in translating science into policy. The task force must be able to respond rapidly and effectively, so data must be exchanged and integrated quickly as they emerge. Thus, when the need occurs, outbreak-management teams can be formed and targeted at a specific outbreak in a defined area of the world. These teams should consist of task-force representatives as well as local experts and policy-makers from the countries affected.

The viability of such an approach is clearly illustrated by the WHO teams formed to deal with the severe acute respiratory syndrome (SARS) outbreaks. Although the achievements of these teams were impressive, the integration between their activities could have been better, and better use could have been made of pre-existing human and animal health networks.

For the current $\mathrm{H} 5 \mathrm{~N} 1$ virus outbreaks, similar WHO teams have been established, but they probably suffer from the same limitations. Given the large geographical area in which the $\mathrm{H} 5 \mathrm{~N} 1$ virus has become endemic, and the greater potential for rapid virus spread, an efficient, effective, outbreak-management team strategy, with centralized guidance, is urgently needed. As key international players related to human and animal health, the WHO, the OIE and the United Nations Food and Agriculture Organization (FAO) are in the unique position to provide the political goodwill and to endorse the proposed integrated approach to the problem of avian flu, which does not respect national borders.

The immediate duties of our proposed task force are fourfold. First, to gain insight into the global picture of flu, taking into account temporal and geographical variation of the virus, in the different species involved (wild birds, poultry, humans, other domestic animals such as pigs, horses and cats, and other wild animals such as seals, cetaceans and tigers). Second, to prioritize research and integrate knowledge of different disciplines on influenza virus infections. Third, to advance intervention strategies for animal outbreaks and human cases. And fourth, to translate knowledge into policy advice, emphasizing the integration of human and animal health strategies.

In dealing with a specific virus outbreak in a defined geographical area, the task force's outbreak teams would focus on: geographical distribution and the species involved in the outbreak; gaining detailed knowledge of the different aspects of the viruses and their interactions with hosts; assessing the risk of spread; and advising on the best options for intervention.

\section{Cost-effectiveness}

Early detection and rapid response to avian flu at the global level will greatly reduce the direct and indirect costs of dealing with a full-blown flu outbreak. For example, in the H7N7 outbreak in the Netherlands and the $\mathrm{H} 5 \mathrm{~N} 1$ outbreaks in Thailand and Vietnam in 2003, the agricultural costs alone were estimated to be US $\$ 348$ million, US $\$ 880$ million and US $\$ 120$ million, respectively. These costs do not take into account the costs of human sickness and death, or the damage done to other areas of the economy, such as tourism.

By contrast, we estimate that the cost of setting up and operating a global task force would be less than US $\$ 1.5$ million a year. The costs of a global human flu pandemic would far exceed the costs estimated to have resulted from the 2003 SARS pandemic, and dwarf the amount of money needed for effective containment and prevention.

Ron Fouchier, Thijs Kuiken, Guus Rimmelzwaan and Albert Osterhaus are at the National Influenza Centre and Department of Virology, Erasmus Medical Centre Rotterdam, Dr Molewaterplein 50, 3015GE Rotterdam, The Netherlands.

1. Stohy, K \& Esveld, M.Science 306, 2195-2196(2004).

2. Kuiken, T,Fouchier, R. A, Rimmelzwaan G. F. \& Osterhaus, A. D.Curr. Opin. Biatechnol. 14, 641-646 (2008)

3. Fouchier, R. A. et al Proc. Natl Acad Sci. USA 101,1356-1361 (2004)

4. Fouchier, R. A, Rimmelzwaan, G. F, Kuiken, T. \& Osterhaus, A. D. Curr. Opin. Infect. Diss 18, 141-146 (2005). 5. www.who int/tosr/disease/avian_influenza/country/en 6 de Jong, M. D. etal. N. Engl. J. Med 352, 686-691 (2005).

7. Webster, R.G.Bean, W. L, Gorman, O.T,Chambers, T.M. \& Kawaoka, Y.Micobiol. Rev. 56,152-179 (1992).

8. WHO Lancet $361,1730-1733$ (2003)

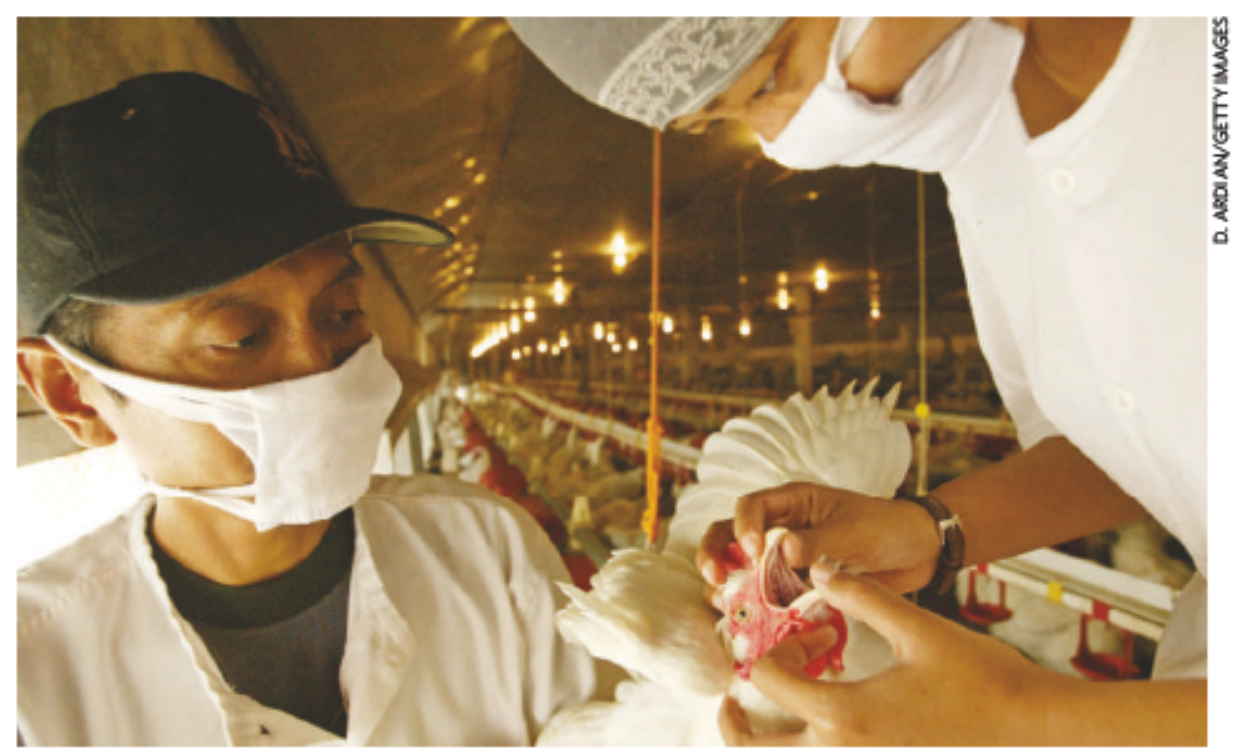

